\section{A Dystroglycan Mutation Associated with Limb-Girdle Muscular Dystrophy}

\author{
Yuji Hara, Ph.D., Burcu Balci-Hayta, Ph.D., Takako Yoshida-Moriguchi, Ph.D., \\ Motoi Kanagawa, Ph.D., Daniel Beltrán-Valero de Bernabé, Ph.D., \\ Hülya Gündeşli, M.S., Tobias Willer, Ph.D., Jakob S. Satz, Ph.D., \\ Robert W. Crawford, B.S., Steven J. Burden, Ph.D., Stefan Kunz, Ph.D., \\ Michael B.A. Oldstone, M.D., Ph.D., Alessio Accardi, Ph.D., Beril Talim, M.D., \\ Francesco Muntoni, M.D., Haluk Topaloğlu, M.D., Pervin Dinçer, Ph.D., \\ and Kevin P. Campbell, Ph.D.
}

SUMMARY

Dystroglycan, which serves as a major extracellular matrix receptor in muscle and the central nervous system, requires extensive O-glycosylation to function. We identified a dystroglycan missense mutation (Thr192 $\rightarrow$ Met) in a woman with limb-girdle muscular dystrophy and cognitive impairment. A mouse model harboring this mutation recapitulates the immunohistochemical and neuromuscular abnormalities observed in the patient. In vitro and in vivo studies showed that the mutation impairs the receptor function of dystroglycan in skeletal muscle and brain by inhibiting the post-translational modification, mediated by the glycosyltransferase LARGE, of the phosphorylated O-mannosyl glycans on $\alpha$-dystroglycan that is required for high-affinity binding to laminin.

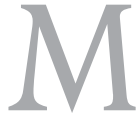

USCULAR DYSTROPHIES ARE GENETIC DISEASES CHARACTERIZED BY weakness and progressive degeneration of skeletal muscle. The transmembrane protein dystroglycan, which is ultimately cleaved into an $\alpha$ and a $\beta$ component, is a key link between the cytoskeleton and extracellular-matrix proteins that bear laminin globular domains (e.g., laminin, agrin, and neurexin)., ${ }^{1,2}$ The mucin domain of $\alpha$-dystroglycan is modified with numerous O-linked oligosaccharides that are essential for its normal function as an extracellular-matrix receptor in various tissues, including skeletal muscle and brain. ${ }^{2,3}$ Hypoglycosylation of $\alpha$-dystroglycan and a consequent reduction of $\alpha$-dystroglycan binding to extracellularmatrix proteins are observed in patients with the Walker-Warburg syndrome, muscleeye-brain disease, Fukuyama-type congenital muscular dystrophy, congenital muscular dystrophy types $1 \mathrm{C}$ and $1 \mathrm{D}$, and limb-girdle muscular dystrophy $2 \mathrm{I}^{2}{ }^{2}$ To date, genes encoding six putative and known glycosyltransferases (POMT1, POMT2, POMGnT1, LARGE, FKTN, and FKRP) have been shown to be responsible for approximately $50 \%$ of cases of these diseases, which are collectively referred to as secondary dystroglycanopathies ${ }^{4-11}$ and which often feature brain abnormalities as well as muscular dystrophy. Despite recent advances in our understanding of the molecular mechanisms underlying secondary dystroglycanopathies, it remains unclear whether dystroglycan is the only target of these enzymes or whether other substrates contribute to the pathogenesis of these diseases.

Dystroglycan is known to be modified with several classes of oligosaccharides: N-glycans, mucin-type O-glycans, and O-mannosyl glycans. ${ }^{2}$ The O-mannosyl gly-
From the Departments of Molecular Physiology and Biophysics (Y.H., T.Y-M., M.K., D.B-V.B, T.W., J.S.S., R.W.C., A.A., K.P.C.), Neurology (K.P.C.), and Internal Medicine (K.P.C.), and the Howard Hughes Medical Institute (K.P.C.), University of lowa Roy J. and Lucille A. Carver College of Medicine, lowa City; the Departments of Medical Biology (B.B.-H., H.G., P.D.), Pediatric Pathology (B.T.), and Pediatric Neurology (H.T.), Faculty of Medicine, Hacettepe University, Sihhiye, Ankara, Turkey; the Molecular Neurobiology Program, Helen L. and Martin S. Kimmel Center for Biology and Medicine at the Skirball Institute of Biomolecular Medicine, New York University Medical School, New York (S.J.B.); the Institute of Microbiology, University Hospital Center and University of Lausanne, Lausanne, Switzerland (S.K.); the Department of Immunology and Microbial Science, Scripps Research Institute, La Jolla, CA (M.B.A.O.); and the Dubowitz Neuromuscular Centre, University College London Institute of Child Health, London (F.M.). Address reprint requests to Dr. Campbell at the University of lowa Roy J. and Lucille A. Carver College of Medicine, 285 Newton Rd., 4283 CBRB, lowa City, IA 52242, or at kevin-campbell@uiowa.edu.

Drs. Hara and Balci-Hayta contributed equally to this article.

N EnglJ Med 2011;364:939-46 Copyright @) 2011 Massachusetts Medical Society. 
cans are thought to be responsible for dystroglycan's ligand-binding activity because the POMT1POMT2 protein complex catalyzes the transfer of mannose to serine or threonine residues by means of an O-linkage. ${ }^{2}$ We recently reported that maturation of $\alpha$-dystroglycan to its laminin-binding form requires a novel biosynthetic pathway involving phosphorylation on O-mannosyl glycans and that LARGE is crucial for further modification of phosphorylated O-mannosyl glycans on $\alpha$-dystroglycan. ${ }^{12}$

Here we report a missense mutation in the dystroglycan-encoding gene, DAG1, in a patient with limb-girdle muscular dystrophy and cognitive impairment. Our studies revealed that this substitution interferes with LARGE-dependent maturation of phosphorylated O-mannosyl glycans on $\alpha$-dystroglycan and leads to the disease-causing defect of $\alpha$-dystroglycan binding to laminin.

\section{METHODS}

\section{CLINICAL INFORMATION}

The patient initially received a diagnosis of limb-girdle muscular dystrophy with mental retardation. ${ }^{13}$ Clinical data regarding this patient have been reported elsewhere. ${ }^{14}$ (See also the Supplementary Appendix, available with the full text of this article at NEJM.org.) This study was approved by the institutional review board at Hacettepe University, and written informed consent was obtained from the patient's guardians.

\section{BIOCHEMICAL ANALYSIS}

Biochemical analyses, including Western blotting, laminin-binding assays, and protein pull-down assays, were carried out as previously described. ${ }^{3,15}$ (For details about the cell-culture and biochemical methods used, see the Supplementary Appendix.)

\section{MOUSE MODEL}

Protocols for generating a knock-in mouse model harboring a mutation equivalent to that in our patient, as well as animal care and animal procedures, were approved by the University of Iowa Animal Care and Use Committee (see the Supplementary Appendix).

\section{RESULTS}

We studied a Turkish patient with a relatively mild form of muscular dystrophy accompanied by cog- nitive impairment (see Case 2 in Dinçer et al. ${ }^{14}$ ). Although immunofluorescence analysis showed that $\alpha$-dystroglycan was hypoglycosylated in this patient, no mutation was found in genes previously implicated in the pathogenesis of secondary dystroglycanopathies. ${ }^{14}$ Since the dystroglycanencoding gene, DAG1, is a plausible candidate gene, we investigated whether our patient harbored a causative mutation therein. Sequencing of the coding and noncoding regions of DAG1 revealed a homozygous C-to- $\mathrm{T}$ missense mutation at position c. 575 , causing substitution of a methionine for a threonine at amino acid residue $192(\mathrm{~T} 192 \rightarrow \mathrm{M})$ of the protein (Fig. 1A). Both parents were heterozygous carriers of this mutated allele, and allele segregation in the family was consistent with recessive disease inheritance (Fig. 1B). The mutated threonine residue is located in the $\alpha$-dystroglycan $\mathrm{N}$-terminal and is highly conserved across species (Fig. 1C).

We first assessed the effect of the newly identified $\alpha$-dystroglycan mutation in vitro, with respect to expression, post-translational processing, or both. To this end, we transiently expressed the full-length wild-type and T192 $\rightarrow$ M dystroglycan proteins in dystroglycan-null myoblasts. Western blotting with an antibody that recognizes functionally glycosylated $\alpha$-dystroglycan (IIH6) ${ }^{3,15}$ revealed considerably lower immunoreactivity for T192 $\rightarrow$ M than for wild-type dystroglycan when similar amounts of protein were expressed (Fig. 1D). Biotinylation analysis revealed that the cellsurface expression of T192 $\rightarrow M$ and wild-type dystroglycan was similar (Fig. 1A in the Supplementary Appendix). Thus, in myoblasts, the T192 $\rightarrow$ M substitution affects neither protein localization nor protein stability, but it does impair posttranslational modification. A laminin-binding assay confirmed that the T192 $\rightarrow$ M mutation considerably reduces the effectiveness of dystroglycan as a laminin receptor (Fig. 1D, and Fig. 1B and 1C in the Supplementary Appendix).

We next assessed the effect of the T192 $\rightarrow$ M mutation on functional modification of $\alpha$-dystroglycan in vivo by introducing a T190 $\rightarrow$ M mutation (which corresponds to the human T192 $\rightarrow \mathrm{M}$ mutation) into the mouse Dag1 gene (Fig. 2 in the Supplementary Appendix). Histologic analysis revealed hallmarks of muscular dystrophy, such as centrally nucleated fibers, in the mutant mice (Fig. 2A, and Fig. 3A and 4 in the Supplementary Appendix). Immunofluorescence and biochemical analyses showed that the mutated 


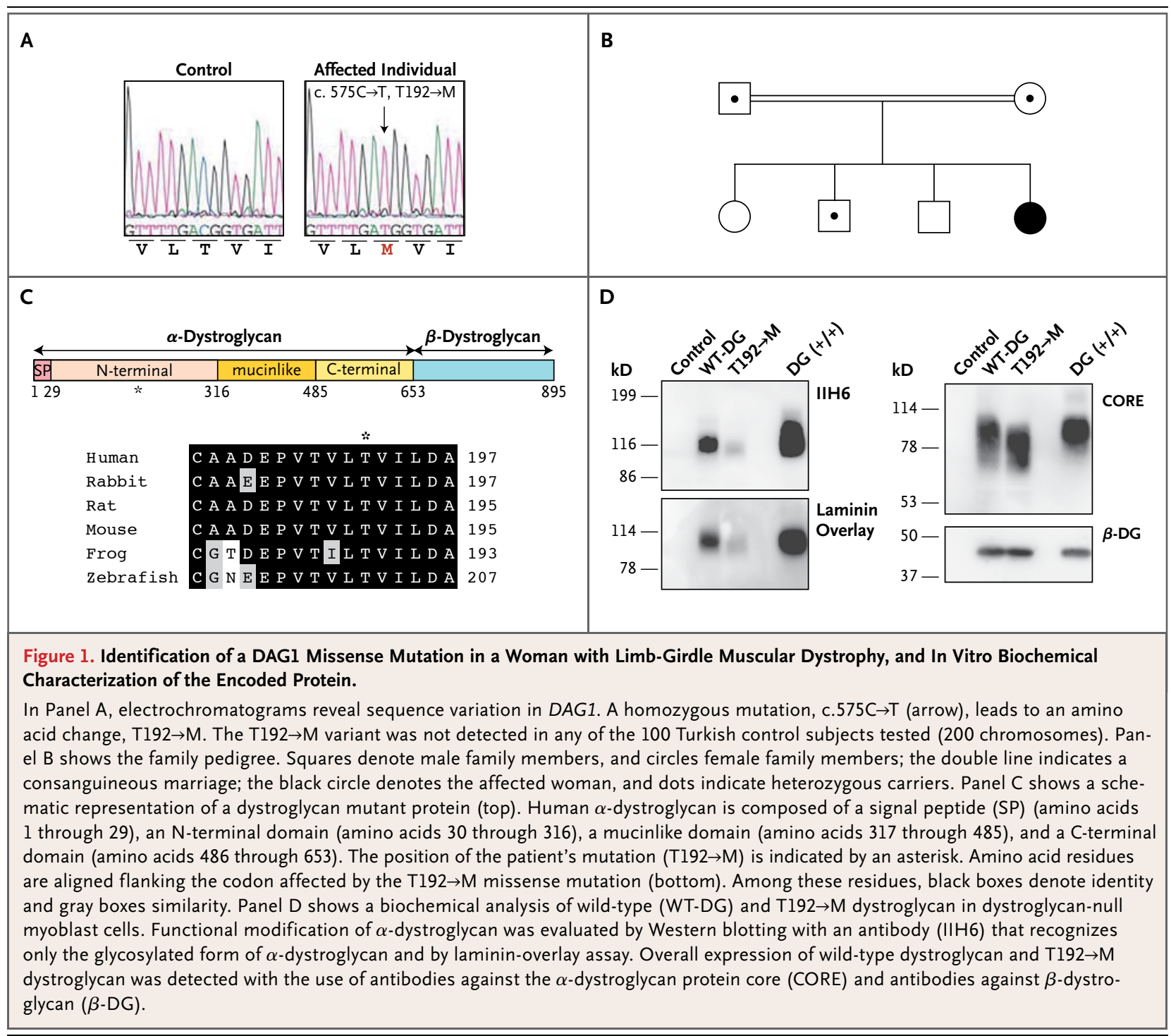

$\alpha$-dystroglycan was expressed at the skeletalmuscle sarcolemma but that both ligand-binding activity and IIH6 reactivity at this location were considerably decreased (Fig. 2B, and Fig. 3B and 5 in the Supplementary Appendix). Analysis of the uptake in the diaphragm of the membrane-impermeable Evans blue dye revealed disruption of the skeletal-muscle sarcolemma in the T190 $\rightarrow$ M mice after exercise (Fig. 2C). The mutant mice also had marked impairment of exercise performance (e.g., reduced forelimb grip strength) (Fig. 2D). Mice heterozygous for the T190 $\rightarrow$ M mutation did not have obvious muscle defects at the histologic level, despite a slight reduction in functional glycosylation of $\alpha$-dystroglycan (Fig. 4 and 6 in the Supplementary Appendix). Thus, the T190 $\rightarrow$ M muta- tion caused skeletal-muscle abnormalities in mice that were consistent with those observed in our patient.

In addition to its role in skeletal muscle, dystroglycan is crucial for maintaining the structure and function of the brain and neuromuscular junctions. ${ }^{3,16-18}$ Western blot and laminin-overlay analyses revealed that laminin-binding activity was significantly diminished in the brains of mice with the T190 $\rightarrow$ M mutation (Fig. 3A). Morphologic analysis of the neuromuscular junctions revealed that the T190 $\rightarrow$ M mutation interfered with their maturation; on staining of diaphragm samples with $\alpha$-Bungarotoxin conjugated with Alexa Fluor 488 (Invitrogen), which tightly binds acetylcholine receptors, the neuromuscular junctions 
in wild-type animals formed the expected pretzellike structure, whereas those in the $\mathrm{T} 190 \rightarrow \mathrm{M}$ mice were round and immature (Fig. 3B and 3C, and Fig. 7 in the Supplementary Appendix). Consistent with impaired neuromuscular-junction maturation was the observation that the mutant mice had marked deficiencies in assays of neuromuscular function - for example, a reduction in retention time on a rotating-rod device (Fig. 3D). Furthermore, although no structural abnormality was evident in the brains of the T190 $\rightarrow \mathrm{M}$ mice (an observation that was consistent with the results on magnetic resonance imaging [MRI] of the patient's brain), these mice had abnormal hindlimb clasping, a phenotype common to mouse models featuring neurologic impairment (Fig. 8 in the Supplementary Appendix). Collectively, these results provide strong evidence that the mutation in the patient caused neurologic impairment as well as muscular dystrophy as a consequence of impaired $\alpha$-dystroglycan post-translational modification.

Dystroglycan is also expressed in cardiac muscle, where its function as an extracellular-matrix receptor is important for limiting activity-induced myocardial damage. ${ }^{19}$ Given that some patients with mutations in FKTN and FKRP have dilated cardiomyopathy, ${ }^{20,21}$ we analyzed cardiac tissue from $\mathrm{T} 190 \rightarrow$ M mice. We observed no obvious signs of any pathological abnormality, such as fibrosis or uptake of Evans blue dye after exercise (Fig. 9 in the Supplementary Appendix). Moreover, in contrast to the observed effects in brain and skeletal muscle, laminin-binding activity was affected only minimally in the hearts of T190 $\rightarrow$ M mice (Fig. 3A). Thus, it appears that normal cardiac function is maintained in T190 $\rightarrow$ M knock-in mice and that the effects of the mutation on functional modification of $\alpha$-dystroglycan are tissue-dependent.

Mutations in LARGE have been identified in patients with the Walker-Warburg syndrome or congenital muscular dystrophy type $1 \mathrm{C}$, as well as in mice with myodystrophy (Large ${ }^{m y d}$ ). ${ }^{9,10,22} \mathrm{We}$ recently found that LARGE is essential for phosphorylated O-mannosyl glycans to mature into a form capable of binding laminin. ${ }^{12} \mathrm{We}$ investigated the glycosylation status of $\mathrm{T} 190 \rightarrow \mathrm{M}$ $\alpha$-dystroglycan by subjecting the protein to treatment with cold aqueous hydrofluoric acid, which cleaves phosphoester linkages, and to inorganic metal-affinity chromatography, which captures monoester-linked phosphorylated compounds. Hy-
Figure 2 (facing page). Biochemical and Physiological Skeletal-Muscle Phenotypes of Disease in Gene-Targeted Mice with the Same Dystroglycan Mutation as the Patient. Panel A shows immunofluorescence and histologic analyses of T190 $\rightarrow$ M skeletal-muscle (iliopsoas) sections prepared when the animals were 21 weeks of age. Serial sections were stained with antibodies against glycosylated $\alpha$-dystroglycan (IIH6), the $\alpha$-dystroglycan core (CORE), and $\beta$-dystroglycan ( $\beta$-DG). Histologic abnormalities in the sections were evaluated by means of hematoxylin and eosin ( $\mathrm{H}$ and $\mathrm{E})$ staining. White arrowheads indicate centrally nucleated fibers (scale bar, $50 \mu \mathrm{m})$. Panel B shows a representative biochemical analysis of wild-type (WT) and T190 $\rightarrow \mathrm{M} \alpha$-dystroglycan isolated from wheat-germ agglutinin-enriched homogenates of skeletal muscle. Both Western blotting and laminin-overlay assays were carried out. Functional modification of $\alpha$-dystroglycan was impaired by the T190 $\rightarrow$ M mutation in vivo, as shown by the dramatic reduction in $1 \mathrm{IH} 6$ immunoreactivity and laminin binding in the absence of major differences in CORE and $\beta$-DG immunoreactivity. Panel $C$ shows the uptake of Evans blue dye in the diaphragm after exercise in wildtype and $\mathrm{T} 190 \rightarrow \mathrm{M}$ mice at 8 weeks of age. Panel D shows the whole-mouse grip-strength measurements, in gram-force ( $\mathrm{g}-\mathrm{F})$, for the $\mathrm{T} 190 \rightarrow \mathrm{M}$ mice and their wild-type littermates. The $P$ value was obtained with the use of Student's t-test.

drofluoric acid treatment clearly reduced the molecular weight of $\mathrm{T} 190 \rightarrow \mathrm{M} \alpha$-dystroglycan, and the beads on chromatography failed to capture T190 $\rightarrow M \alpha$-dystroglycan, although they readily bound $\alpha$-dystroglycan in samples from Largemyd animals (Fig. 10 in the Supplementary Appendix). These results suggest that T190 $\rightarrow \mathrm{M} \alpha$-dystroglycan bears phosphorylated O-mannosyl glycans but that these moieties are not sufficiently mature to confer laminin-receptor function on $\alpha$-dystroglycan.

Our findings suggest that the T192 $\rightarrow \mathrm{M}$ mutation affects LARGE-dependent modification of $\alpha$-dystroglycan. A previous study showed that the dystroglycan $\mathrm{N}$-terminal serves as a binding site for LARGE, an interaction that is essential for $\alpha$-dystroglycan's laminin-binding activity. ${ }^{15} \mathrm{An}$ in silico model suggested that the side chain of the methionine residue could affect the protein surface structure ${ }^{23}$ (Fig. 11A in the Supplementary Appendix); accordingly, we hypothesized that the T192 $\rightarrow$ M mutation abrogates the dystroglycanLARGE interaction owing to a conformational change in the dystroglycan N-terminal. A pulldown assay with Fc-tagged $\alpha$-dystroglycan mutants showed that although the wild-type pro- 


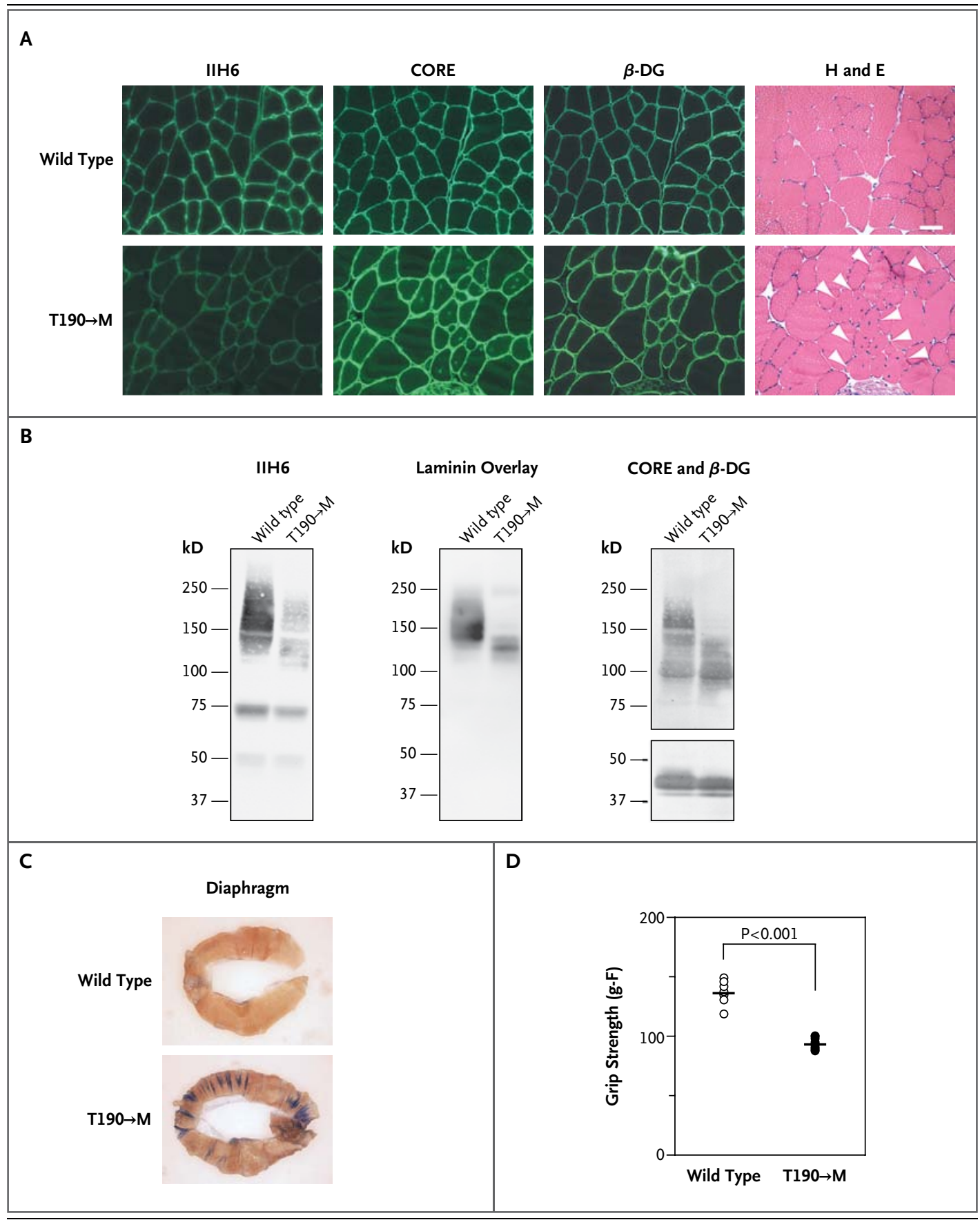

tein bound to LARGE, variants harboring the T192 $\rightarrow$ M mutation did not (Fig. 11B and 11C in the Supplementary Appendix). These findings support the notion that the $\mathrm{T} 192 \rightarrow \mathrm{M}$ mutation in dystroglycan dramatically impairs the interaction between $\alpha$-dystroglycan and LARGE, leading to incomplete post-translational modification of $\alpha$-dystroglycan.

$\overline{\text { DISCUSSION }}$

Although genes responsible for O-glycosylationdependent muscular dystrophy (i.e., dystroglycanopathy) have been identified, the molecular mechanisms whereby mutations therein lead to impaired skeletal-muscle and brain function remain elusive - in part because defects in dystro- 


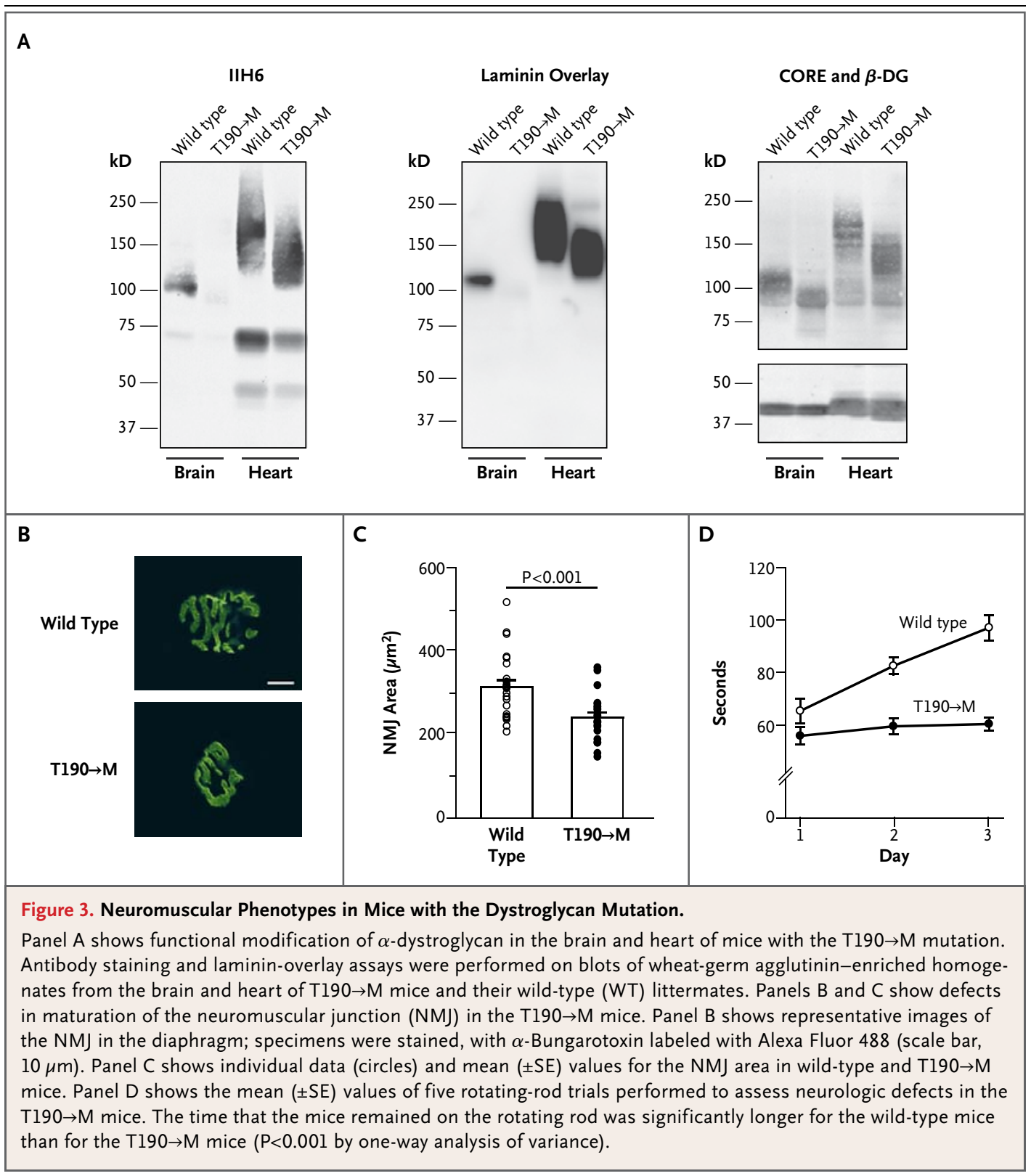

glycan itself have not been reported. Here we describe such a case, which represents a new disease class (i.e., primary dystroglycanopathy). The T192 $\rightarrow$ M mutation in this case caused deficiencies in $\alpha$-dystroglycan glycosylation and a marked reduction in $\alpha$-dystroglycan's ability to bind extracellular-matrix components. Furthermore, our knock-in mouse model harboring the patient's mutation recapitulates the phenotype of secondary dystroglycanopathies in humans, supporting the view that dystroglycan is the main - and probably only - protein that is subject to the glycosylation abnormalities that cause muscular dystrophy.

Patients with dystroglycanopathy have a broad spectrum of disease phenotypes, with or without brain involvement. Limb-girdle muscular dystrophy with mental retardation was diagnosed in our patient according to the classification system established by Godfrey et al. ${ }^{13}$ Biochemical findings from analyses of mouse T190 $\rightarrow$ M show that laminin-binding activity is considerably reduced (Fig. 1 and 2), suggesting that residual glycosylation may account for the relatively mild 
muscle phenotype, as compared with the findings in patients with other congenital muscular dystrophies.

Our patient had severe cognitive impairment, yet MRI revealed no gross structural abnormality in the brain. ${ }^{14}$ In more severe cases of human dystroglycanopathy, as well as in mice with a brain-specific Dag1 knockout, structural as well as functional brain abnormalities have been documented..$^{3-6,9,10,16}$ Thus, it may be that residual laminin binding by human T192 $\rightarrow$ M dystroglycan is sufficient for the development of the cerebral layer but not for the establishment of normal synaptic physiology. It remains unclear how dystroglycan contributes to synaptic physiology; however, reports that synaptic components possessing laminin globular domains play a fundamental role in establishing and maintaining synaptic function $^{24,25}$ suggest that a reduction in the affinity of dystroglycan for synaptic proteins could be responsible for the neurologic phenotypes observed in the patient described here.

Our biochemical analyses show that the T192 $\rightarrow$ M mutation impairs maturation of a specific modification of phosphorylated O-mannosyl glycans on $\alpha$-dystroglycan (Fig. 10 in the Supplementary Appendix). In the heart, T190 $\rightarrow$ M $\alpha$-dystroglycan maintains substantial lamininbinding activity despite a reduction in molecular weight consistent with that in skeletal muscle and brain (Fig. 2 and 3A), and this remaining activity may contribute to preserved cardiac function (Fig. 8 in the Supplementary Appendix). The tissue variability in levels of $\alpha$-dystroglycan's functional glycosylation remains unexplained, but there are several possible explanations (e.g., tissue-specific protein complexes might increase the affinity between dystroglycan and LARGE, or the enzymatic activity of LARGE might be enhanced in heart tissue). Further investigation will be required to delineate the tissue-specific mechanisms ensuring that phosphorylated O-mannosyl residues of $\alpha$-dystroglycan mature into lamininbinding glycans.

In conclusion, we found a pathogenic missense mutation in DAG1 that selectively impairs further modification of phosphorylated O-mannosyl glycans on $\alpha$-dystroglycan, leading to neuromuscular abnormalities. Our findings constitute evidence for the inclusion of DAG1 in the list of genes whose mutations cause muscular dystrophy and cognitive impairment in humans.

\begin{abstract}
Disclosure forms provided by the authors are available with the full text of this article at NEJM.org.

Supported in part by grants from the Senator Paul D. Wellstone Muscular Dystrophy Cooperative Research Center (1U54NS053672, to Dr. Campbell), the Hacettepe University Research Fund (03.02.101.009, TÜBİTAK Project SBAG-1774, to Dr. Dinçer), the National Institutes of Health (AI45927, to Dr. Oldstone), the National Institute of Diabetes and Digestive and Kidney Diseases (P30 DK 54759, to the University of Iowa Gene Transfer Vector Core), and the Muscular Dystrophy Campaign and Institute of Child Health Biomedical Research Centre (to Dr. Muntoni). Dr. Campbell is an investigator at the Howard Hughes Medical Institute.

We thank Harry Schachter, Michael Anderson, Michael Henry, and Charles Harata for their critical comments; Matthew Goddeeris, Colleen Campbell, Holly Harper, Sally Prouty, Mary Anderson, Keith Garringer, and members of the Campbell laboratory for their scientific contributions; Baoli Yang and Eileen Sweezer (University of Iowa Gene Targeting Core Facility) for generating the $\mathrm{T} 190 \rightarrow \mathrm{M}$ dystroglycan knock-in mice; Lydia Sorokin (Münster University) for the antibody against the laminin $\alpha 1$ chain (clone no. 317); and the University of Iowa Gene Transfer Vector Core for adenovirus purification.
\end{abstract}

\section{REFERENCES}

1. Ibraghimov-Beskrovnaya O, Ervasti JM, Leveille CJ, Slaughter CA, Sernett SW, Campbell KP. Primary structure of dystrophin-associated glycoproteins linking dystrophin to the extracellular matrix. Nature 1992;355:696-702.

2. Barresi R, Campbell KP. Dystroglycan: from biosynthesis to pathogenesis of human disease. J Cell Sci 2006;119:199 207.

3. Michele DE, Barresi R, Kanagawa M, et al. Post-translational disruption of dystroglycan-ligand interactions in congenital muscular dystrophies. Nature 2002; 418:417-22.

4. Beltrán-Valero de Bernabé $\mathrm{D}$, Currier $\mathrm{S}$, Steinbrecher A, et al. Mutations in the $O$-mannosyltransferase gene POMT1 give rise to the severe neuronal migration disorder Walker-Warburg syndrome. Am J Hum Genet 2002;71:1033-43.

5. van Reeuwijk J, Janssen $M$, van den Elzen C, et al. POMT2 mutations cause $\alpha$-dystroglycan hypoglycosylation and Walker-Warburg syndrome. J Med Genet 2005;42:907-12.

6. Yoshida A, Kobayashi K, Manya H, et al. Muscular dystrophy and neuronal migration disorder caused by mutations in a glycosyltransferase, POMGnT1. Dev Cell 2001;1:717-24.

7. Kobayashi K, Nakahori Y, Miyake M, et al. An ancient retrotransposal insertion causes Fukuyama-type congenital muscular dystrophy. Nature 1998;394:388-92.

8. Brockington $M$, Blake DJ, Prandini P, et al. Mutations in the fukutin-related protein gene (FKRP) cause a form of congenital muscular dystrophy with secondary laminin $\alpha 2$ deficiency and abnormal glycosylation of $\alpha$-dystroglycan. Am J Hum Genet 2001;69:1198-209.

9. Longman $\mathrm{C}$, Brockington $\mathrm{M}$, Torelli S, et al. Mutations in the human LARGE gene cause MDC1D, a novel form of congenital muscular dystrophy with severe mental retardation and abnormal glycosylation of $\alpha$-dystroglycan. Hum Mol Genet 2003; 12:2853-61.

10. van Reeuwijk J, Grewal PK, Salih MA, et al. Intragenic deletion in the LARGE gene causes Walker-Warburg syndrome. Hum Genet 2007;121:685-90.

11. Mercuri E, Messina S, Bruno C, et al. 
Congenital muscular dystrophies with defective glycosylation of dystroglycan: a population study. Neurology 2009;72: 1802-9.

12. Yoshida-Moriguchi T, Yu L, Stalnaker $\mathrm{SH}$, et al. O-mannosyl phosphorylation of alpha-dystroglycan is required for laminin binding. Science 2010;327:88-92.

13. Godfrey C, Clement E, Mein R, et al. Refining genotype phenotype correlations in muscular dystrophies with defective glycosylation of dystroglycan. Brain 2007; 130:2725-35.

14. Dinçer P, Balci B, Yuva Y, et al. A novel form of recessive limb girdle muscular dystrophy with mental retardation and abnormal expression of $\alpha$-dystroglycan. Neuromuscul Disord 2003;13:771-8.

15. Kanagawa M, Saito F, Kunz S, et al. Molecular recognition by LARGE is essential for expression of functional dystroglycan. Cell 2004;117:953-64.

16. Moore SA, Saito F, Chen J, et al. Dele- tion of brain dystroglycan recapitulates aspects of congenital muscular dystrophy. Nature 2002;418:422-5.

17. Côté PD, Moukhles H, Lindenbaum $\mathrm{M}$, Carbonetto S. Chimaeric mice deficient in dystroglycans develop muscular dystrophy and have disrupted myoneura synapses. Nat Genet 1999;23:338-42.

18. Nishimune H, Valdez G, Jarad G, et al Laminins promote postsynaptic maturation by an autocrine mechanism at the neuromuscular junction. J Cell Biol 2008; 182:1201-15.

19. Michele DE, Kabaeva Z, Davis SL, Weiss RM, Campbell KP. Dystroglycan matrix receptor function in cardiac myocytes is important for limiting activityinduced myocardial damage. Circ Res 2009;105:984-93.

20. Murakami T, Hayashi YK, Noguchi S et al. Fukutin gene mutations cause dilated cardiomyopathy with minimal muscle weakness. Ann Neurol 2006;60:597-602.
21. Mercuri E, Brockington M, Straub V, et al. Phenotypic spectrum associated with mutations in the fukutin-related protein gene. Ann Neurol 2003;53:53742.

22. Grewal PK, Holzfeind PJ, Bittner RE, Hewitt JE. Mutant glycosyltransferase and altered glycosylation of $\alpha$-dystroglycan in the myodystrophy mouse. Nat Genet 2001;28:151-4.

23. Bozic D, Sciandra F, Lamba D, Brancaccio $\mathrm{A}$. The structure of the $\mathrm{N}$-terminal region of murine skeletal muscle $\alpha$-dystroglycan discloses a modular architecture. J Biol Chem 2004;279:44812-6.

24. Südhof TC. Neuroligins and neurexins link synaptic function to cognitive disease. Nature 2008;455:903-11.

25. Sato S, Omori Y, Katoh K, et al. Pikachurin, a dystroglycan ligand, is essential for photoreceptor ribbon synapse formation. Nat Neurosci 2008;11:923-31.

Copyright (c) 2011 Massachusetts Medical Society.

NEW NEJM APPLICATION FOR IPHONE

The NEJM Image Challenge app brings a popular online feature to the smartphone. Optimized for viewing on the iPhone and iPod Touch, the Image Challenge app lets you test your diagnostic skills anytime, anywhere. The Image Challenge app randomly selects from 300 challenging clinical photos published in NEJM, with a new image added each week. View an image, choose your answer, get immediate feedback, and see how others answered.

The Image Challenge app is available at the iTunes App Store. 